Trauma Berufskrankh 2008 10

[Suppl 2]:226-229

DOI 10.1007/s10039-008-1421-x

Online publiziert: 29. Juni 2008

(c) Springer Medizin Verlag 2008

\author{
C. Jacobs · A. Moghaddam • C. Wölfl • P. von der Linden · A. Wentzensen • \\ G. Zimmermann \\ BG-Unfallklinik Ludwigshafen, Klinik für Unfallchirurgie und Orthopädie \\ an der Universität Heidelberg, Ludwigshafen
}

\title{
Wachstumshormone und BMP
}

Eine ausbleibende oder verzögerte Frakturheilung stellt in der Unfallchirurgie noch immer ein häufiges und schwer lösbares Problem dar. Eine in bis $\mathrm{zu} 40 \%$ auftretende Knochenbruchheilungsstörung bei Patienten mit gewissen Risikofaktoren sowie bei $10 \%$ aller Frakturen wurde bereits mehrfach beschrieben. Der Entwicklung einer Pseudarthrose kommt aufgrund der durch sie verursachten Verlängerung der Behandlungsdauer neben der Belastung für den Patienten eine nicht unerhebliche volkswirtschaftliche Bedeutung zu. Bekannte operative Verfahren bei der Ausbildung einer Pseudarthrose sind:

- die Dynamisierung eines Marknagels,

- der Wechsel auf einen aufgebohrten

Nagel oder die Plattenosteosynthese sowie

- die Eigenknochentransplantation.

Wesentlich aufwändiger sind bei frustranem Heilungsverlauf die dann noch verbleibenden operativen Möglichkeiten einer Segmentresektion sowie des Segmenttransports, welche mit entsprechend erhöhte Morbiditätsraten und Risiken bei keinem sicheren Heilungserfolg einhergehen.

Bei atrophen Pseudarthrosen wurden bereits Defizite bestimmter Serumspiegel von Knochenwachstumsfaktoren nachgewiesen. $\mathrm{Zu}$ diesen über 80 bekannten Zytokinen gehören u. a. TGF $\beta$ („transforming growth factor $\beta$ “), FGF („fibroblast growth factor") sowie BMP-7 („,bone morphogenetic protein 7 “) und BMP2. Die BMP gehören zur Superfamilie des TGF $\beta$ und scheinen bei der Steuerung der Frakturheilung eine zentrale Rolle zu spie- len. Seit 2001 können als zugelassene Therapieoption für verzögert heilende sowie offene Unterschenkelfrakturen 2 Wachstumsfaktoren eingesetzt werden:

- BMP-7 bei verzögerter Frakturheilung und

- BMP-2 bei offenen Unterschenkelfrakturen.

Eine Unterstützung der Frakturheilung durch Konzentrationserhöhung der Knochenwachstumsfaktoren erscheint sinnvoll und notwendig und wurde anhand einzelner Studien bereits untersucht.

\section{Knochenbruchheilung}

Die körpereigene Knochenneubildung und -reparatur können prinzipiell auf dreierlei Weisen erfolgen, durch

- Osteogenese,

- Osteokonduktion und

- Osteoinduktion.

Osteogenese. Hierunter wird die Synthese neuen Knochens aus überlebenden Präosteoblasten und Osteoblasten nach Trauma oder Implantation des Autografts verstanden.

Osteokonduktion. Als osteokonduktiv werden Materialien bezeichnet, die ein Gerüst oder Gitternetz zum Einwachsen von Knochen bilden. Dies beinhaltet die Differenzierung und Reifung von Osteoprogenitorzellen innerhalb des Transplantats sowie dessen Kalzifizierung und Vaskularisierung. Idealerweise wird das Implantat schließlich vollständig durch neuen Knochen ersetzt.
Osteoinduktion. Sie beschreibt die Knochenneubildung durch aktive Rekrutierung pluripotenter undifferenzierter Wirtszellen, die sich in Chondro- und Osteoblasten differenzieren.

Voraussetzung für die Fähigkeit des Knochens zur Regeneration sind die fortlaufende Differenzierung induzierbarer Mesenchymzellen (IOPC) und die Proliferation determinierter Osteoblastenvorläuferzellen (DOPC). Mediatoren des Knochenwachstums sind Wachstums- und osteoinduktive Faktoren, wobei Wachstumsfaktoren wie IGF-1 und 2 (,insulin like growth factor"), TGF $\beta_{1}, \mathrm{TGF} \beta_{2}$ und TGF $\beta_{3}$, PDGF („platelet derived growth factor"), aFGF („acidic fibrolast growth factor") und bFGF („basic fibrolast growth factor") eine wichtige Bedeutung zukommt. Sie haben auf ortsständiges Knochengewebe einen regulierenden und proliferationsfördernden Effekt. Da Wachstumsfaktoren die entstehenden oder schon im Knochen vorhandenen Osteoprogenitorzellen beeinflussen, ist ihre Wirksamkeit bei großen knöchernen Defekten begrenzt. Gemeinsam mit anderen Zytokinen werden diese Proliferations- und Differenzierungsvorgänge durch BMP eingeleitet, weshalb sie zur Gruppe der osteoinduktiven Proteine gehören (• Abb. 1).

Innerhalb der ersten Minuten und Stunden wird eine inflammatorische Reaktion in Gang gesetzt, nach Stunden bis Tagen kommt es zu einer Chemotaxis und Mitosis von Stammzellen mit Produktion extrazellulärer Knochenmatrix. Nach Tagen bis Wochen findet anschließend ein Remodelling der Frakturzone mit lokaler Angioneogenese statt. 


\section{Studie}

\section{Ziel}

Ziel der Studie war, die Wirksamkeit der BMP bei der Frakturheilung zu untersuchen und mit Frakturheilungen ohne Einsatz von Knochenwachstumsfaktoren zu vergleichen.

Bei besseren Heilungsraten nach BMPEinsatz, insbesondere bei verzögerten Knochenbruchheilungen, könnten die zusätzliche Anwendung der Knochenwachstumsfaktoren ein neues Behandlungskonzept darstellen und - bei höheren Heilungschancen - gleichzeitig die Behandlungskosten verringert werden.

\section{Aufbau}

Seit Mitte 2002 wird BMP-7 in unserem Haus klinisch angewendet. Zum Einsatz kommt es bei Patienten mit mehreren Therapieversuchen bei atrophen Pseudarthrosen des Tibiaschafts. Entsprechend der Zulassungsstudie ist seine Anwendung in Europa in der Behandlung von Tibiapseudarthrosen nur nach mindestens einer erfolglosen Spongiosaplastik zugelassen. Als Vergleichsgruppe diente ein möglichst einheitliches und ausreichend großes Kollektiv an Patienten mit erstmaliger Spongiosaplastik. Anhand der knöchernen Durchbauung nach 4 Monaten sollte die Wirksamkeit der ersten Spongiosaplastik mit der Anwendung von BMP-7 nach multiplen Voroperationen verglichen werden. Aufgrund der eingeschränkten Einsatzzulassung von BMP-7 sind Vergleiche mit Therapieerfolgen der Spongiosaplastik an einem identischen Patientenkollektiv nicht möglich.

Mit dem von uns gewählten Vergleich (erstmalige Spongiosaplastik vs. multiple Voroperationen und Anwendung von BMP-7) hat zumindest das Patientenkollektiv der BMP-7-Gruppe keine besseren Voraussetzungen.

\section{Patienten}

Bei einer verzögerten Frakturheilung erhielten im Zeitraum von 01/1995-12/2002 82 Patienten erstmalig eine Spongiosaplastik und bildeten die Vergleichsgruppe. Bei 26 Patienten wurde zwischen 05/2002

Trauma Berufskrankh 2008 · 10[Suppl 2]:226-229 DOI 10.1007/s10039-008-1421-x

(c) Springer Medizin Verlag 2008

\section{Jacobs · A. Moghaddam · C. Wölfl · P. von der Linden · A. Wentzensen · G. Zimmermann Wachstumshormone und BMP}

\section{Zusammenfassung}

BMP („,bone morphogenetic proteins") gehören zur Superfamilie des TGF $\beta$ (,transforming growth factor $\beta^{\prime \prime}$, transformierender Wachstumsfaktor $\beta$ ) und scheinen bei der Steuerung der Frakturheilung eine zentrale Rolle zu spielen. Seit 2001 können als zugelassene Therapieoption für verzögert heilende und offene Unterschenkelfrakturen 2 Wachstumsfaktoren eingesetzt werden, BMP-7 bei verzögerter Frakturheilung und BMP-2 bei offenen Unterschenkelfrakturen. Ziel der vorliegenden Studie war, die Wirksamkeit der BMP bei der Frakturheilung zu untersuchen und derjenigen ohne Einsatz von Knochenwachstumsfaktoren gegenüber zu stellen. Verglichen wurden Patienten, die nach mehreren Therapieversuchen bei atrophen Pseudarthrosen des Tibiaschafts mit BMP-7 behandelt wurden, mit einem möglichst ein-

\section{Growth hormones and BMP}

\section{Abstract}

BMP (bone morphogenetic proteins) belong to the TGF $\beta$ superfamily and appear to play a central role in fracture healing. Two growth factors have been in use since 2001 as an approved therapy option for the delayed healing of fractures or open lower leg fractures: BMP-7 for delayed fracture healing and BMP2 for open lower leg fractures. The aim of the present study was to examine the effectiveness of the BMP in fracture healing and compare fracture healing without the use of bone growth factors. Patients who were treated with BMP-7 following repeated attempts to treat atrophic pseudoarthrosis of the tibia were compared with a large standardized heitlichen und ausreichend großen Kollektiv an Patienten mit erstmaliger Spongiosaplastik. Patienten, bei denen BMP-7 angewendet worden war, zeigten nach 4 Monaten eine signifikant bessere knöcherne Durchbauung des Frakturbereichs anhand radiologischer Untersuchungen. Um die Überlegenheit dieses Verfahrens nachzuweisen und somit auch eine generelle Behandlungsempfehlung aussprechen zu können, sind jedoch Untersuchungen an größeren Patientenkollektiven und prospektiv auch in der Vergleichsgruppe erforderlich.

\section{Schlüsselwörter}

„bone morphogenetic protein".

Transformierender Wachstumsfaktor $\beta$. Frakturheilung $\cdot$ Pseudarthrose $\cdot$ Knöcherne Durchbauung collective of patients with first-time spongiosa plastic. Patients receiving BMP-7 showed significantly better bone fusion in the fracture area at radiological examination at four months. In order to prove the advantages of this procedure, as well as to be able to recommend it as a treatment modality, further studies on larger patient collectives and prospective control groups are needed.

\section{Keywords}

Bone morphogenetic proteins - Transforming growth factor $\beta$. Fracture healing .

Pseudoarthrosis · Bone fusion 


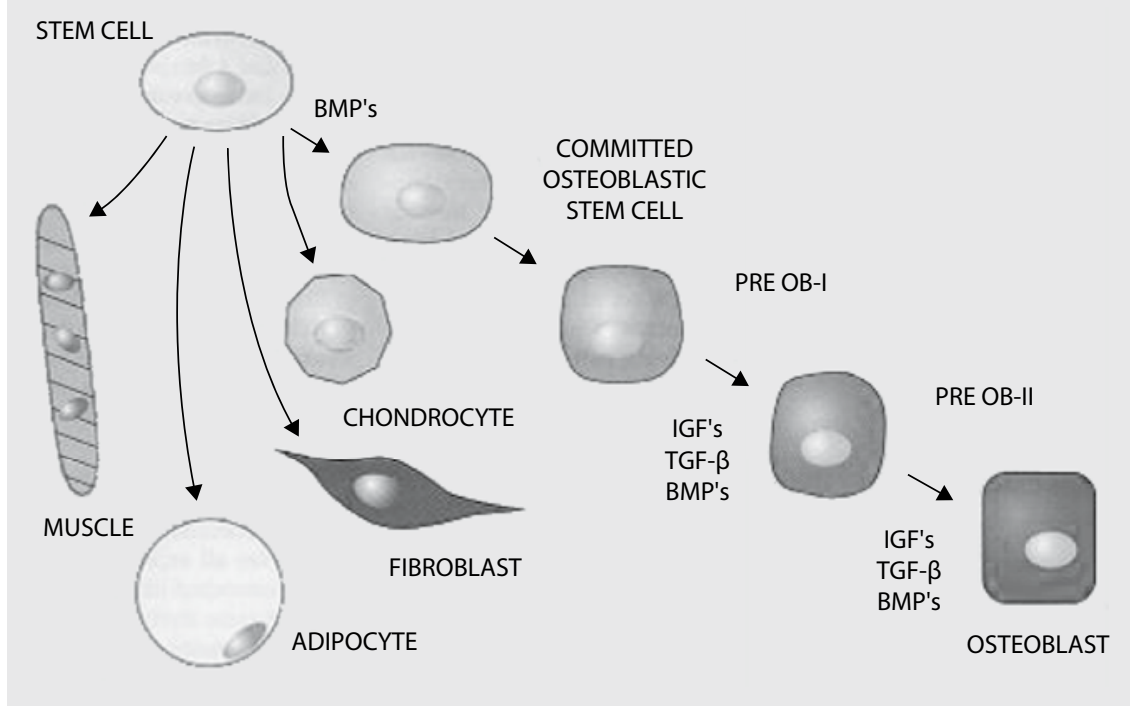

Abb. $1 \Delta$ Ablauf der Knochenbruchheilung

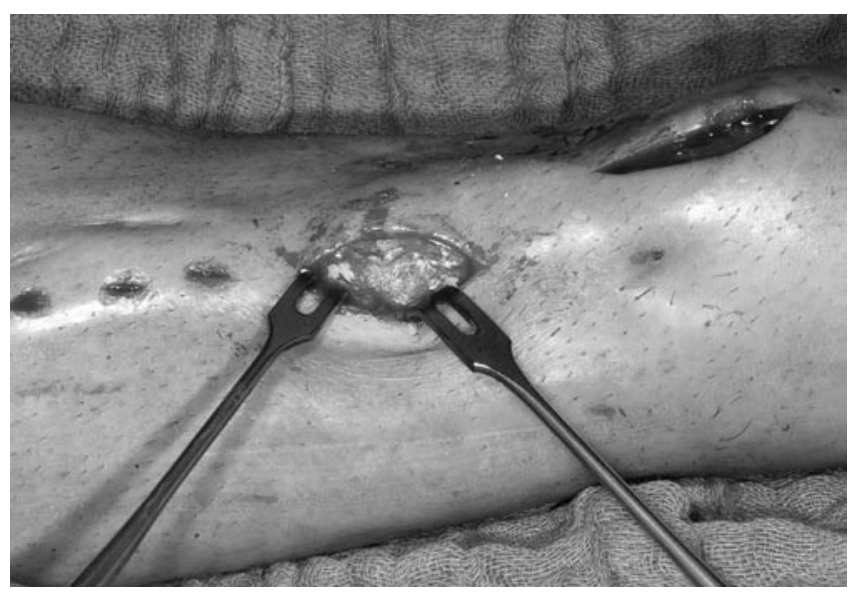

Abb. $2<$ Implantation von BMP-7

und 06/2005 BMP-7 nach durchschnittlich 3 erfolglosen Revisionen implantiert. Sie bildeten die Studiengruppe. Die knöcherne Durchbauung des Frakturbereichs nach 4 Monaten wurde anhand radiologischer Untersuchungen evaluiert.

\section{Operationstechnik}

Die debridierte Pseudarthrose wird so weit angefrischt, dass blutende Knochenenden vorliegen. Nach Abschluss aller operativen Maßnahmen wie Osteosynthese, Jetlavage und ggf. zusätzlicher Spongiosaentnahme sollte BMP-7 als letzter Schritt implantiert werden. Auf eine Dränage direkt an der Implantationsstelle muss verzichtet werden, da sonst das Protein eingesaugt werden könnte. Um den Wachstumsfaktor sicher vor Ort zu halten, decken wir die Implantationsstelle mit einem Hämostyptikum $\left(\right.$ Tachoseal $\left.^{\circledR}\right)$ ab.
Eine Implantationseinheit BMP-7 besteht in ihrer Darreichungsform aus 3,5 mg Protein als Pulver an $1 \mathrm{~g}$ Kollagen 1 gebunden (- Abb. 2). Vor der Implantation muss sie noch mit 3-5 ml Patientenblut oder Kochsalzlösung angereichert werden, um eine Art „nasser Sand“ zu erhalten. Die Menge an implantiertem Protein übertrifft bei weitem die normalerweise physiologisch vorhandene Konzentration im Frakturbereich, sodass durch eine Erhöhung der lokalen Dosis keine höhere Wirksamkeit zu erwarten ist. Die simultane Implantation von 2 Einheiten BMP-7 stellt zudem die zulässige Höchstmenge dar.

\section{Ergebnisse}

In unserer Klinik überblicken wir bisher etwa 100 Anwendungen von BMP mit einer Erfolgsquote von insgesamt 77\% bei mehrfach voroperierten Patienten. In der vorliegenden Vergleichstudie von 26 Patienten unserer Klinik, welche mit BMP-7 bei Tibiafrakturen therapiert wurden und durchschnittlich 3 Voroperationen erhalten hatten, lag die Ausheilungsrate ohne weitere Maßnahme bei $92 \%$.

Bei der Vergleichsgruppe zeigte sich bei 24 Patienten (28\%) nach erfolgter Spongiosaplastik ohne BMP-7 keine knöcherne Durchbauung. Im Gegensatz dazu kam es bei der Gruppe mit BMP-7 nur bei 2 Patienten (8\%) zu keiner knöchernen Durchbauung des Frakturbereichs. Die ermittelten Ergebnisse zeigten eine signifikant höhere Heilungsrate mit $\mathrm{p}=0,025$ für Patienten, bei denen BMP-7 angewendet worden war. Trotz des Patientenkollektivs mit der schlechteren Ausgangssituation in der Studiengruppe mit zahlreichen Voroperationen konnte bei diesen ein besserer Verlauf erzielt werden.

\section{Diskussion}

Knochenwachstumsfaktoren und insbesondere BMP stimulieren die Frakturheilung. Physiologisch werden sie beim Frakturereignis lokal freigesetzt und begleiten den Prozess der Frakturheilung bis hin zum Remodelling des Knochens. Auch wenn inzwischen etwa 80 Wachstumsfaktoren und andere Faktoren bekannt sind, welche essenziell den Knochenregenerationsprozess begleiten, sind momentan nur 2 von diesen zur klinischen Anwendung zugelassen und ihre Wirksamkeit in prospektiven, randomisierten Studien nachgewiesen.

In unserer Studie konnte eine besserer Heilungsrate bei Patienten mit Anwendung von BMP-7 trotz der zahlreichen Voroperationen nachgewiesen werden. Der Einsatz von BMP-7 bei Pseudarthrosen langer Röhrenknochen ist zusammenfassend erfolgreich und komplikationsfrei. Eine Datenbank über möglichst alle Anwendungen in Deutschland sollte künftig verlässliche Angaben über oben genannte Punkte liefern, um fundierte Aussagen über die breite klinische Anwendung dieses Knochenwachstumsfaktors treffen zu können.

In der aktuellen Literatur finden sich derzeit noch keine Untersuchungen von BMP-7 an größeren Patientenkollektiven 
zur Behandlung von mehrfach voroperierten Tibiapseudarthrosen. In der hier vorliegenden Studie konnte eine signifikant bessere Wirksamkeit von BMP-7 als durch Eigenknochentransplantation trotz der schlechteren Ausgangssituation der Patienten festgestellt werden. Auch wenn aufgrund der geringeren Gruppengröße der Studiengruppe das Ergebnis statistisch gesehen grenzwertig ist, kann man zumindest von einer vergleichbaren Wirksamkeit beider Verfahren ausgehen. Dies gewinnt auch vor dem Hintergrund der geringeren Komplikationsraten bei BMP-7-Implantation an Bedeutung. Um die Überlegenheit eines Verfahrens nachzuweisen und somit auch eine generelle Behandlungsempfehlung aussprechen zu können, sind jedoch noch Untersuchungen an größeren Patientenkollektiven notwendig und prospektiv auch in der Vergleichsgruppe durchzuführen.

Entscheidend für die Therapie mit BMP-7 sind auch die Erfolgsrate und sowie die Kosten im Vergleich zur Spongiosaplastik. Leider liegen sowohl zur Effizienz als auch zur Kostenrechnung keine harten Daten vor. In einer retro- und prospektiven Studie werteten wir die Erfolgsrate einer Eigenknochentransplantation in unserer Klinik aus. Hierbei wurden isoliert Tibiaschaftfrakturen mit verzögerter Frakturheilung und anschließender Spongiosaplastik untersucht. Als erfolgreich wurde die Spongiosaplastik gewertet, wenn die Fraktur ohne weitere Maßnahmen klinisch und radiologisch ausheilte, als erfolglos, falls eine erneute Intervention aufgrund ausbleibender Frakturheilung erforderlich war. Als Vergleich dienten Patienten, welche an unserer Klinik aufgrund einer Pseudarthrose an der Tibia mit BMP-7 therapiert wurden. Die Erfolgskriterien waren identisch mit denen der Eigenspongiosagruppe. Es zeigte sich eine signifikant $(p=0,025)$ höhere Erfolgsrate für BMP-7 trotz höherer Voroperationsquote gegenüber der Eigenspongiosagruppe.

Volkswirtschaftlich ergab sich ebenfalls ein Vorteil für BMP-7. Die Kosten für eine Einheit BMP-7 betragen momentan zwischen 4000 und 5000 EUR. Die Kosten für eine Spongiosaplastik, welche durch einen Einsatz von BMP-7 mitunter vermieden werden kann, liegen geschätzt zwischen 500 (reine operationstechnische Kosten) und 4000 EUR (volkswirtschaftliche Kosten inklusive Komplikationen, ambulante Behandlung und ggf. Dauerinvalidität). Wesentlich höher sind aber die volkswirtschaftlichen Folgekosten einer nicht zeitgerecht heilenden Fraktur. So konnten Sprague u. Bhandari [3] und Heckman u. Sarasohn-Kahn [2] in einer Kostenanalyse zur verzögerten Frakturheilung auch bei Unterschenkelverletzungen zeigen, dass durch nur 16 Wochen Verlängerung des Krankheitsverlaufs zusätzliche volkswirtschaftliche Kosten von fast 82.000 \$ entstehen.

Eine Kostenanalyse für die Behandlung von drittgradig offenen Unterschenkelfrakturen mit BMP-2 liegt von Alt u. Heissel [1] vor. Auf der Basis von über 500 Patienten, welche entweder mit oder ohne BMP behandelt wurden, zeigte sich bei der BMP-Gruppe eine Kosteneinsparung von 5685 EUR/Patient.

\section{Schlussfolgerung}

Die Therapie verzögert heilender Frakturen mit BMP-7 ist erfolgreich und komplikationslos. Es muss davon ausgegangen werden, dass BMP-7 in vielen Fällen wirksamer ist als die Eigenknochentransplantation. Um eine generelle Behandlungsempfehlung aussprechen zu können, bedarf es aber künftig noch weiterer Studien an größeren Patientenkollektiven. Zum jetzigen Zeitpunkt kann anhand unserer Studie jedoch nachgewiesen werden, dass die Behandlung mit BMP-7 zumindest die gleiche Wirksamkeit hat wie die Eigenknochentransplantation, bei jedoch geringerer Morbidität. Somit bildet sie v. a. bei Patienten mit Risikofaktoren eine komplikationsärmere Alternative zur Spongiosaplastik.

\section{Korrespondenzadresse}

PD Dr. G. Zimmermann

BG-Unfallklinik Ludwigshafen,

Klinik für Unfallchirurgie und Orthopädie

an der Universität Heidelberg,

Ludwig-Guttmann-Straße 13,

67071 Ludwigshafen

Zimmermann@BGU-Ludwigshafen.de

Interessenkonflikt. Der korrespondierende Autor gibt an, dass kein Interessenkonflikt besteht.

\section{Literatur}

1. Alt V, Heissel A (2006) Economic considerations for the use of recombinant human bone morphogenetic protein-2 in open tibial fractures in Europe: the German model. Curr Med Res Opin [Suppl 1] 22: $\mathrm{S} 19-22$

2. Heckman JD, Sarasohn-Kahn J (1997) The economics of treating tibia fractures. The cost of delayed unions. Bull Hosp Jt Dis 56: 63-72

3. Sprague S, Bhandari M (2002) An economic evaluation of early versus delayed operative treatment in patients with closed tibial shaft fractures. Arch Orthop Trauma Surg 122: 315-323 